\title{
Working Memory Capacity, AtTENTION CONTROL, AND Fluid INTELLIGENCE
}

\author{
RichaRd P. HeITZ \\ NASH UnSWORTH \\ RANDALL W. ENGLE
}

Or take the power of attention. Is this wholly, or partly, or not at all the same as intelligence? All three views are widely held in the literature.

Spearman (1927, p. 13)

$\mathrm{T}$ he idea that attention is important to intelligence is not novel. Indeed, Spearman (1927) discussed this issue at length. However, the relationship between attention and intelligence was contemplated even earlier, with some of the first empirical evidence provided by Burt (1909). Binet (1903), the father of intelligence testing, also recognized the importance of attention to general intelligence (Sternberg, 1982). William James (1890/1981), too, wrote that "the number of things we may attend to is altogether indefinite, depending on the power of the individual intellect" (p. 405). However, despite nearly a century of research, the above question still pervades the science of mental ability, and Spearman's comment

AUTHORS' NOTE: This research was supported by Grants F49620-97-1 and F49620-93-1-0336 from the Air Force Office of Scientific Research. Correspondence concerning this article should be addressed to Richard P. Heitz or Randall W. Engle, School of Psychology, Georgia Institute of Technology, 654 Cherry Street, NW, Atlanta, GA 30332. E-mail: richard.heitz@psych.gatech.edu, gtg039d@prism.gatech.edu, or randall.engle @ psych.gatech.edu. 


\section{2 • HANDBOOK OF UNDERSTANDING AND MEASURING INTELLIGENCE}

appears to be as true today as it was in 1927. However, converging evidence from both experimental and factor-analytic methodologies is beginning to support the notion that attention is one of the major determinants of general fluid intelligence-the ability to reason abstractly in novel environments and situations.

The difficulty in studying the complex attention-intelligence relationship is partly one of semantics. Both constructs are latent factors, in that they elude direct observation and must be measured indirectly. Thus, one must cautiously select measurement tools that are most sensitive to the constructs of interest. Because one's theory of attention and intelligence guides the selection of measures, observed correlations will partially depend on one's underlying theory. For this reason, we take particular care in defining our conception of attention and intelligence. In the sections that follow, we do just that, and in the interest of our guiding theory, we show that one's ability to control attention is related to one's potential for abstract reasoning.

There are three general types of theories in the psychometric study of intelligence. The first stresses the idea of "positive manifold"- the observation that individuals who score high on one intelligence test tend to score high on other intelligence tests. This idea, originated by Spearman (1904, 1927), suggests that there is a single intellectual ability on which people differ. At the other end of the spectrum are theories proposing no single psychometric factor but instead a variety of specific intelligences (Thurstone, 1938). Still others hold a somewhat intermediate view (e.g., Sternberg, 1985). One such view that has become widely accepted is the hierarchical model of Cattell (1943, 1963). Cattell's viewpoint is that $g$ is actually composed of two components: general-crystallized (Gc) and general-fluid (Gf) intelligence (see also Carroll, 1993). Accordingly, Gf reflects one's ability to reason abstractly and perform well in novel environments, whereas Gc corresponds to abilities and knowledge accumulated over time, as might be measured by tests of factual knowledge. Evidence that Gf consistently relates to performance in higher-order cognitive tasks (e.g., Engle, Kane, \& Tuholski, 1999) leads us to believe that attention exerts its strongest effect on Gf, and we limit our discussion to this construct.

This aside, there is also disagreement as to what constitutes psychometric $g$ in factoranalytic methodology. Commonly, $g$ emerges as the first principal component or the highest order factor extracted from a battery of broad cognitive tasks; lesser factors are generally interpreted in a manner consistent with those measures showing the highest loadings (simple structure). But this need not be the case. The extraction of $g$ is just as much a part of theory as it is of statistical methodology. For example, Cattell (1943) notes that, using different statistical methodologies, one can support either Spearman's $g$ factor or Thurstone's specific intelligences. It is important, therefore, to have sound theoretical grounds for proposing the existence of $g$, Gf, Gc, or any other manifestation of intellectual factors.

\section{Measuring Intelligence AND ATtENTION}

Exactly what constitutes a good measure of intelligence has been, particularly in earlier research, rather post hoc. Criticizing practices in intelligence research, Cattell (1943) admonishes test developers who validate their measure by correlating it with other intelligence tests. This, he says, "takes the view that intelligence is what has been measured by past intelligence tests" (p. 159). This circular logic appears in much of the current literature, at least in some form. In examining the relationship between working memory (WM) and intelligence (at least in experimental research), it is not uncommon that researchers use a single intelligence measure (e.g., the Raven Progressive Matrices) as the criterion (e.g., Brewin \& Beaton, 2002). This begs the following question: What is special about the Raven test that other measures lack? The answer to this question lies in the fact that the measure consistently loads highly on $g$ and appears to be quite sensitive to individual differences (Marshalek, Lohman, \& Snow, 1983). Although this is appropriate for small-scale experimental research, more sensitive tests involve the extraction of a Gf latent factor. For this reason, we have used the Raven test alone 
in experimental research and, in addition, have included multiple measures of Gf in our factoranalytic studies, reported below.

A similar problem exists in defining attention. William James's (1890/1981) adage that "everyone knows what attention is" is certainly true on the level of introspection, but deriving a definition that all can agree on proves difficult. Some attest that attention serves only to maintain information through activation (Anderson, Reder, \& Lebiere, 1996), some renounce activation in favor of inhibition (Hasher \& Zacks, 1988), and others remain agnostic, treating attention as simply controlled cognition (e.g., Schneider \& Shiffrin, 1977). We take a more comprehensive view. Certainly, there is a dissociation between controlled and automatic processing, with the former being effortful and subject to some type of (attentional) capacity limitation. And the manner in which control operates is likely due to specialized mechanisms, such as activation and inhibition. Our stance, then, is that attentional control is a voluntary, effortful cognitive act that serves to maintain information through activation of relevant brain circuitry, inhibit the irrelevant and distracting information that impinges on us at any one time, and suppress prepotent response tendencies that are task irrelevant. It is important to keep in mind that this is qualitatively different from a situation in which attention is automatically captured, such as by a flashing light or an unexpected sound. Note that this theory is far reaching. By our definition, any type of information that may be lost over time due to decay or interference must be maintained through attentional control. This may take the form of a grocery list while driving in busy traffic, or it may be a response production such as "Look away from the flashing cue" when the flashing cue tends to reflexively capture attention or the control of a socially unacceptable implicit attitude to prevent it from being expressed in publicly observable behavior.

Such a stance is consistent with a number of so-called "varieties of attention." As noted by a number of researchers (e.g., Parasuraman, 1998), attention serves different types of functions depending on task demands. One variety of attention comes into play during sustained attention tasks, such as vigilance. In such a task, participants must maintain attention in the service of detecting an infrequent target. Those who are able to maintain attention to the task at hand are more able to quickly respond to the onset of targets. The attention required in a vigilance task is somewhat different than that required on, say, a divided-attention task, in which participants must perform two tasks simultaneously. Still another variety of attention is manifest in selective-attention tasks, in which participants are presented multiple sources, or channels, of information, and they must select one and disregard another. Although it might be argued that attention serves different roles in vigilance, divided-attention, and selectiveattention tasks, we would argue that what is common to all is the control of attention. In other words, attention can be put to use in a variety of ways, but the act of voluntarily employing attention always relies on capacity-limited executive control. For example, we find that both selective-attention tasks, such as dichotic listening (Conway, Cowan, \& Bunting, 2001), and divided-attention tasks (often a cognitive task incorporating a secondary load; e.g., Kane \& Engle, 2000) reliably differentiate individuals rated high or low in attentional control ability, but tasks that can be performed automatically do not (Kane, Bleckley, Conway, \& Engle, 2001; Tuholski, Engle, \& Baylis, 2001). From this, it is apparent that individuals who differ in their ability to use controlled attention may be dissociated using a variety of paradigms. In other words, working memory capacity (WMC) and, by our logic, attention control are not task dependent but are a factor in any task that requires attention control (Turner \& Engle, 1989).

One activity that does not differentiate individuals is automatic attention capturecertainly a special case of attentional phenomena. Here, an innate mechanism directs attention, effortlessly and without volition, to some stimulus such as the sudden appearance of some object in the periphery or any feature such as flicker that suggests movement. This is certainly an adaptive response, as suddenly appearing objects may be hazardous or helpful, predator or prey. Although automatic attentional capture does not require control, resisting attention capture by a salient event does require control (Kane et al., 2001). That is, executive control 


\section{4 • HANDBOOK OF UNDERSTANDING AND MEASURING INTELLIGENCE}

is important for the endogenous prevention of exogenous capture from both internal and external distractors.

To understand the attention-intelligence issue, we must first explain how we measure these two constructs, as the validity of a latent factor is only as good as the manifest variables comprising it. In the next section, we review microanalytic research suggesting that tasks thought to tap WMC reliably reflect one's ability to control attention. Following this, we describe macroanalytic studies that show a significant relationship between WMC and fluid intelligence. As we illustrate in this chapter, it is apparent that WMC and fluid intelligence are in some way related but clearly not isomorphic. We submit that the relationship is due to the ability to control attention - an aspect of cognition tapped by both WMC and fluid intelligence tests. In a final section, we consider such alternative explanations as processing speed and task complexity.

\section{Microanalytic Studies OF Working MEMORY And Controlled AtTention}

It is important to realize that the central executive component of the working memory system is an attentional construct. This is clear in recent models proposed by Baddeley (e.g., 1996), but it is also evident in earlier models. In particular, Atkinson and Shiffrin (1968) noted that information held in the short-term store (STS) decays over time, unless control processes are invoked to refresh these traces using rehearsal. Thus, attention needs to be directed to information held online to keep it active. Admittedly, this view is quite different from theories of immediate memory focusing on the number of units of information that can be maintained over time (e.g., G. A. Miller, 1956). But, as we contend, WMC is not about a number or an amount per se. Using extreme group methodology, we have observed numerous dissociations between individuals rated high and low in WMC that simply cannot be explained in terms of a limitation based on a number of units. The differences are intrinsically qualitative rather than quantitative. In other words, although we measure
WMC quantitatively by the number of items recalled on complex span task, the scores on such measures reflect controlled-attention ability rather than the number of "units" of information that can be held in a short-term store. Accordingly, we propose that WMC is an ability reflecting the extent to which an individual is able to control attention, particularly in situations involving interference from competing information, activated representations, or task demands.

Before citing evidence for the controlled attention view of WMC, it is necessary to make clear exactly how WMC is assessed and the rationale for this particular methodology. In line with the view that WM consists of both domainspecific storage buffers and a domain-general central executive mechanism, tasks that load the central control mechanism should lead to a decrease in the amount of information that can be maintained. In other words, including a heavy processing component should tax the attentional system to such an extent that to-berecalled information is more likely to be lost from domain-specific stores. Dual-task situations are often employed to this end. And, interestingly, dual-task situations appear to capture some ability that is related to g. Fogarty and Stankov (1982) presented individuals with auditory tasks requiring maintenance and/or manipulation of tones or letters. In one condition, participants performed these tasks alone; in another, these tasks were performed as dual tasks. They found that the tasks correlated more strongly when they were components of a dual task than when they were performed alone, suggesting that some additional ability became necessary when performing the two tasks simultaneously that was not required by either task alone. In addition, Fogarty and Stankov examined the first principal component (representing all of the tasks). As Fogarty and Stankov argue, the nature of the tasks employed suggests that the first principal component can be considered a Gf factor. They found that dual tasks load higher on the first principal component than single tasks, suggesting that they were better measures of Gf. In other words, competing tasks showed higher positive manifold than single tasks (Stankov, 1983; but see Stankov, 1989). Recent neuroimaging research strengthens the 
view that dual tasks measure an important executive ability. D'Esposito, Detre, Alsop, and Shin (1995) had participants perform single or dual tasks in a functional magentic resonance imaging (fMRI) scanner. They found that dual tasks elicit marked increases in prefrontal cortex activity, whereas either task performed alone did not. This would be expected given (a) the link between prefrontal cortex and executive control (see Kane \& Engle, 2002, for a review; see also Chapter 9, this volume) and (b) our view that dual-task situations place heavy demands on executive control.

The first widely accepted test of WMC was constructed by Daneman and Carpenter (1980). Known as the reading span task (RSpan), it is inherently a dual task in that it consists of both storage and processing components. In the original RSpan task design, participants were asked to read sets of sentences out loud. After from two to six sentences (each set size presented three times), participants were cued to recall the last word of each. The point at which the participants could accurately recall the final words two out of three times was taken as their WMC. Critically, WMC was significantly correlated with higher-level comprehension measures, such as reading comprehension and the ability to identify a noun referent for a later occurring pronoun. Daneman and Carpenter (1980, 1983; Daneman \& Tardif, 1987) argued that WMC reflects processing efficiency involved in reading. Accordingly, those individuals with efficient reading comprehension processes have available more residual WMC to use for maintaining the to-be-recalled words. Evidence against the processing efficiency idea came from a study using a WMC task in which participants solved arithmetic strings and remembered the word that followed each string. Turner and Engle (1989) found that this operation span (OSpan) ${ }^{1}$ score correlated just as well and often higher with measures of comprehension than those reported by Daneman and Carpenter (1980), indicating that the correlation between WMC and higher-order cognition is not dependent on the similarity of the processing component with the task being predicted; thus, the RSpan-comprehension correlation could not be due to efficiency of reading processes, as Daneman and Carpenter asserted.
Another possible explanation of the correlation between WMC and reading comprehension was that some individuals simply have larger lexicons than others. This hypothesis argued that both complex span and reading comprehension performance are predicated on word knowledge, leading to a correlation. Engle, Nations, and Cantor (1990) reasoned that if this were true, then the WMC-reading comprehension correlation should be strong with low-frequency words but should disappear with high-frequency words. They found that the WMC-comprehension correlation was somewhat stronger with low-frequency words but still very much significant with high-frequency words. From this, Engle et al. (1990) argued that although word knowledge does play some role in the WM span-comprehension correlation, a general WM capacity limitation was the important predictor.

Dual tasks such as RSpan and OSpan appear to measure an important aspect of real-world high-level cognition, as they are found to correlate with such tasks as language comprehension, following directions, vocabulary learning, reasoning, complex learning, and note taking (Engle, Kane, et al., 1999). The critical difference between dual-task situations and a single task, such as simple digit or word span, is that dual tasks require additional processing above and beyond that required by simple span tasks. The extra processing load incurred by these tasks, by hypothesis, occupies the central executive, making it more difficult to refresh traces in WM. However, it might also be that some individuals are simply better at multitasking in dual-task situations, being able to strategically allocate resources toward the primary and secondary components of the task in an effort to maximize span scores. These same individuals may also be more strategic during complex cognitive tasks, leading to a WMC-higher-order cognition correlation. This stance, termed the strategic allocation hypothesis, was evaluated by Engle, Cantor, and Carullo (1992). To evaluate this hypothesis, Engle et al. used a moving window technique to assess the amount of processing given to different segments of the WMC task, with amount of processing operationally defined as viewing time (VT). If high spans are more strategic in resource allocation, one would expect to observe a significant negative correlation between VT on the 


\section{6 • HANDBOOK OF UNDERSTANDING AND MEASURING INTELLIGENCE}

processing component and span score. The authors found that viewing times did not correlate with span scores, leaving the strategic allocation hypothesis untenable.

Tasks such as RSpan and OSpan might appear to reflect individual differences in the number of items that can be maintained in an attention-demanding dual-task situation. However, evidence makes clear that a theory of WMC focusing on a limitation in the number of elements that can be maintained is misguided. Again, the view advanced in the present work is that individual differences in WMC reflect a relatively stable characteristic of an individual's ability to control attention to maintain information in an active or easily accessible state in interference-rich contexts as well as block irrelevant, distracting information.

Evidence for this claim is found in a number of different paradigms. One elegant test is provided by Conway et al. (2001) using the venerable "cocktail party phenomenon." Moray's (1959) dichotic listening paradigm, originally used by Cherry (1953), provided evidence that particularly salient information from an unattended channel is allowed through an attentional filter (see also Wood \& Cowan, 1995). In Moray's classic study, participants were asked to shadow words from a relevant channel while ignoring information in an irrelevant channel. At some point, the participant's own name was spoken in the unattended message. Moray found that approximately $33 \%$ of participants reported hearing their name when it was presented to the unattended ear. Conway et al. reasoned that if working memory capacity is related to the ability to control attention in the service of selecting relevant input and inhibiting irrelevant input, then those rated high in WMC should be less likely to report hearing their own name in the irrelevant channel during a dichotic listening task. The results confirmed that high spans were indeed more proficient in inhibiting the irrelevant channel: Low-span individuals were much more likely $(65 \%)$ to report hearing their own name than were high-span individuals (20\%). This suggests that high-span individuals were indeed better at blocking distractors from the message they were instructed to ignore, whereas low-span individuals were less able to block the distracting information.
Analogous data are provided by Kane et al. (2001), using the anti-saccade task. In these studies, participants were required to make a saccade (an overt eye movement) toward (prosaccade) or away from (anti-saccade) a flashing exogenous cue to identify a briefly presented letter: B, P, or R. Because exogenous cues automatically capture attention, the anti-saccade condition required the inhibition of a prepotent response as well as a controlled saccade in the opposite direction. There are two ways performance is hindered in the anti-saccade condition. The first is through interference: Participants who are not adept at inhibiting the automatic prepotent response will be slow to make a correct saccade in the anti-saccade condition (requiring that one look away from a flashing cue). The second is through a loss in goal maintenance. For example, if the goal ("When flashing left look right") is lost, participants should tend to make a rapid error by looking toward the flashing cue, with their behavior guided by the prepotent response tendency to look toward a flashing stimulus. Following from our theory, it was expected that high- and low-span individuals should not differ in pro-saccade performance but should differ in anti-saccade performance: When an anti-saccade is performed correctly, low spans should be slower, indicating a difficulty in resolving conflict. Low spans should also commit more errors, indicative of goal neglect (De Jong, Berendsen, \& Cools, 1999). And when an error occurs, it should be fast, indicating that the behavior was indeed automatic. These hypotheses were confirmed: High- and low-span participants did not differ in prosaccade performance but did differ in both latency and error rate for anti-saccade performance. And when an error did occur, it was very fast, indicative of goal neglect.

Interestingly, in an additional experiment, Unsworth, Schrock, and Engle (2003) showed that span differences do emerge in a pro-saccade condition if you force individuals to perform them in a controlled manner. Unsworth et al. intermixed pro- and anti-saccade trials within the same block. Each trial began with a symbol that designated whether the trial required a proor anti-saccade. Under this situation, low spans were both slower and more error prone on both pro- and anti-saccade trials. Unsworth et al. 
argue that this manipulation placed a premium on actively maintaining the goal of the task, creating a situation in which both pro- and antisaccade trials must be performed with control.

It would be very difficult to reconcile a theory of WMC based on the maintenance of some number of items with such a finding. That is, if WMC limitations were due to a lack of available "slots" to hold information, it should not play a role in the anti-saccade task. As we argue, the common mechanism affecting both anti-saccade performance and WMC performance is the ability to employ controlled attention. It appears that in the anti-saccade task, span differences in response time (RT) are due to a differential ability to inhibit the reflexive orienting response and execute a planned saccade in the opposite direction-aspects of an ability to exert endogenous control over attention, as we define it. Also in agreement with the controlled attention theory is an apparent inability of low spans to actively maintain the response production "Look away from the flashing cue." Loss of this production may be the locus for span differences in error rate in the anti-saccade condition.

These observations led Engle and colleagues to propose a two-factor theory of executive attention, whereby interference leads to increased latency and goal neglect leads to fast errors. Lending further credence to this theory is a study by Kane and Engle (2003). Participants performed a color-word Stroop task under different proportions of congruency. Previous research (e.g., Logan \& Zbrodoff, 1979) indicates that individuals make more errors on incongruent Stroop trials (saying the word red printed in green ink instead of naming the ink color only) when there is a small proportion of them relative to congruent Stroop trials. One interpretation for this observation is that the repeated presentation of an incongruent trial (such as the word blue presented in red ink) reinforces the goal of the task. Conversely, when incongruent trials are infrequent, participants lose maintenance of the response production "Respond to the color, not the word" because most of the time, they can perform well by relying on relatively automatic word reading. The prediction, then, following from the antisaccade findings, is that if high- and low-span participants differ in their ability to resolve conflict and maintain the goal of the task, they should show greater differences in the Stroop task when incongruent trials are relatively infrequent. This hypothesis was supported. When $75 \%$ of the trials were congruent, highs and lows differed markedly in accuracy rate but not in RT, suggesting that the manipulation increased goal neglect for low spans. However, the Stroop task is also rich in interference, a condition likely to favor high WMC individuals, as previously discussed. When there were few or no compatible trials, WM span groups did not differ in accuracy rate but did differ slightly in RT. That is, when the task goal was continually reinforced by presenting many incongruent trials, low spans were slower to say the color of the ink, presumably because it took them longer to resolve the conflict between the response tendencies to say the word and to say the name of the color. These findings illustrate two important functions of WM: maintenance of goalrelevant information (i.e., name the color and not the word) and dealing with interference through inhibition of prepotent responses (incongruent Stroop trials). In summary, tasks that require controlled attention to maintain information, inhibit distractor information, or block prepotent response tendencies seem to dissociate individuals scoring low or high on dual tasks such as the OSpan or RSpan.

The dissociations between high and low WMC individuals described above employ relatively low-level cognitive tasks. However, these differences are true for higher-level tasks as well. Low spans suffer more than high spans in a proactive interference task (Kane \& Engle, 2000), in a degree-of-fan task (Cantor \& Engle, 1993; Conway \& Engle, 1994), and in a category generation paradigm that requires suppression (Rosen \& Engle, 1997; see also Rosen \& Engle, 1998).

\section{Motivation}

The prevalence and direction of span differences leaves open the possibility that some factor other than differences in ability to control attention differentiates the two groups. Namely, it is often the case that high-span individuals 


\section{8 • HANDBOOK OF UNDERSTANDING AND MEASURING INTELLIGENCE}

outperform low spans; thus, the suggestion that the groups differ along some dimension such as "motivation" cannot be ignored, though there is good evidence against such a view. For example, high- and low-span groups differ in theoretically meaningful and predictable ways: They do not differ in a typical pro-saccade task, in visual search, or in the first trial of a proactive interference task (before interference has built up), as mentioned above. If low spans were simply unmotivated, one would not expect them to perform as well as high spans in any condition.

A thorough test of this explanation was made by Heitz, Schrock, Payne, and Engle (2003). They measured task-evoked pupil dilations while individuals found to be high and low spans (measured using OSpan) performed the RSpan task. Pupillary dilation has been shown to be sensitive to mental effort and processing load (Hess \& Polt, 1964; Kahneman \& Beatty, 1966). Thus, if span groups do differ along a dimension such as motivation, one would expect larger task-evoked pupillary responses from high spans, who may simply expend more effort during task performance. Of course, it could also be that high spans are simply more efficient information processors (e.g., Daneman \& Carpenter, 1980) than low spans, which would predict smaller pupil dilations from high spans. Interestingly, Ahern and Beatty (1979) found that this was true for high- and low-intelligence individuals (high intelligence exhibiting smaller pupil dilations), although there are problems with their methodology (see Heitz et al., 2003).

Heitz et al. (2003) found that although highspan individuals consistently recall more letters correctly than do low spans, they do not exert more mental effort. Specifically, high spans did not exhibit larger pupil dilations during encoding of the letter, while reading the sentence, or, critically, when recalling the letters. ${ }^{2}$ However, pupillary dilation was sensitive to processing load in this study, as there was a linear increase in pupil size as set sizes grew larger. In addition, baseline pupil size measurements, recorded at the beginning of each trial, were strongly correlated with the set size on the previous trial, suggesting that how "hard" one had to work previously affected one's pupil size later. Furthermore, a monetary incentive reliably increased baseline pupil size but in equal amounts for both groups. In other words, monetary incentive encouraged both groups to "try" harder.

The above experimental studies make a strong case for the hypothesis that WMC, as measured by dual-task situations such as OSpan and RSpan, reflect one's ability to control attention. This capability is important when distraction and interference place a premium on maintaining information, particularly in the presence of strong internal or external interference. If we are correct in assuming that the ability to control attention is important to Gf, then a latent factor made up of WM tasks should be strongly related to a latent variable made up of Gf measures. To test this, we turn to factoranalytic methodology.

\section{Macroanalytic Studies of WMC ANd Fluid InTELligence}

One could pose a number of initial questions regarding the relationship between working memory and fluid intelligence that microanalytic research cannot address. First, do the various WMC tasks reflect a common construct? Second, does that construct show construct validity in predicting some other criterion behavior as well as discriminant validity? And third, what is the relationship between WMC and other constructs such as short-term memory (STM)? We argued previously that dual-task situations tap an attentional control ability that is important to higher level cognition. If it is indeed true that WMC tasks tap an ability above and beyond that required by simple span tasks, a structural equation model including separable STM and WM constructs should provide a better fit to the data than a model consisting of a single latent factor. In addition, if these constructs show good discriminant validity, a STM latent factor should not correlate significantly with a Gf factor, but a WM latent factor should.

Obviously, no cognitive measure is "process pure," meaning that no task measures only STM or only WMC. Quite to the contrary, we assert that both simple span tasks and complex span tasks reflect similar constructs but in different proportions. That is, simple span or STM tasks measure, by and large, domain-specific storage 


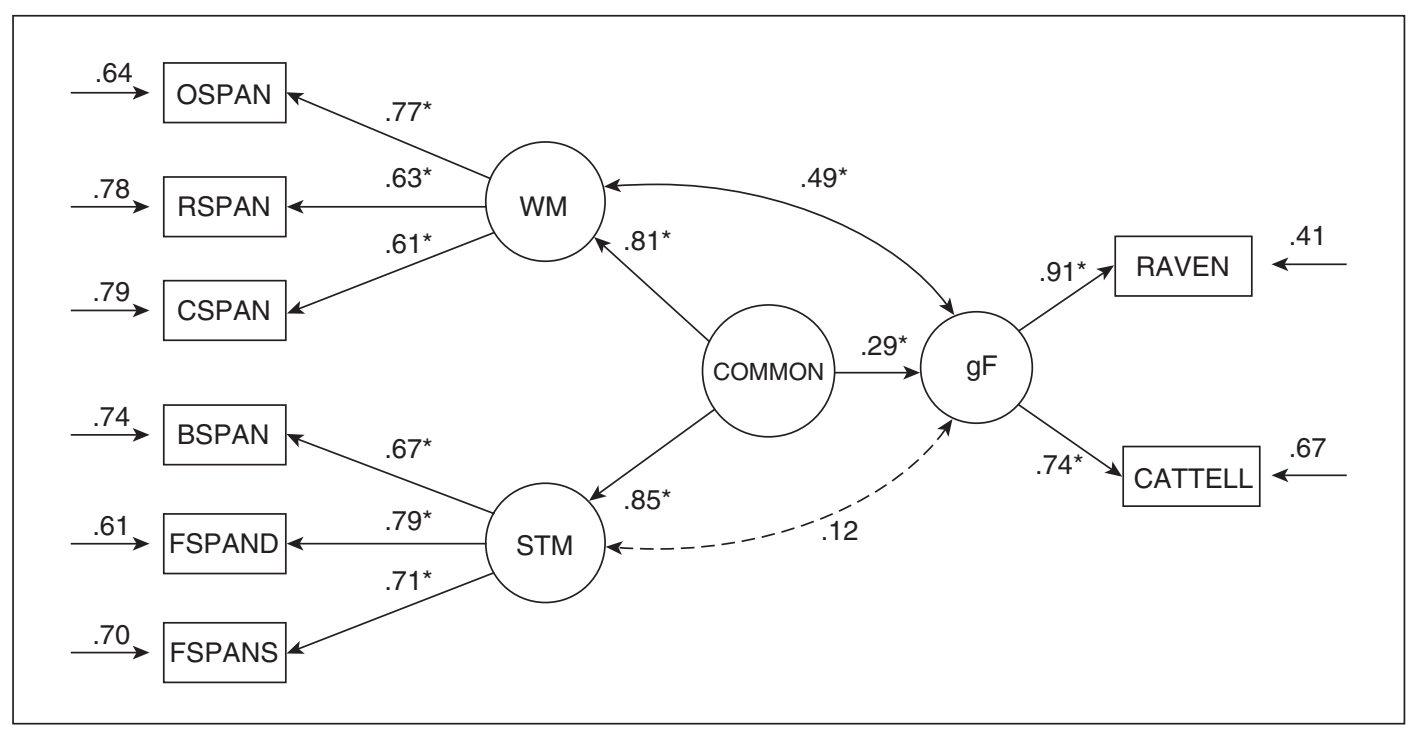

Figure 5.1 Structural Equation Model From Engle, Tuholski, et al. (1999)

Note: The latent WM variable still correlates with the latent Gf variable after partialling the variance due to STM. Boxes represent manifest, or observed, variables; circles represent latent factors. Paths marked with an asterisk are significant at $p<.05 . \mathrm{WM}=$ working memory; $\mathrm{STM}=$ short-term memory; $\mathrm{Gf}=$ general fluid intelligence; OSPAN = operation span; RSPAN $=$ reading span CSPAN $=$ counting span $;$ BSPAN $=$ backwards digit span FSPAND $=$ forward digit span — dissimilar; FSPANS = forward digit span—similar; RAVENS = Raven's Progressive Matrices; CATTELL = Cattell's Culture-Fair Test.

and rehearsal strategies but undoubtedly require some executive ability as well. In contrast, complex span or WMC tasks measure executive attention capability most extensively but also domain-specific storage and rehearsal mechanisms present in the former tasks. For this reason, we expected that although dissociable, STM and WM should be related. Engle, Tuholski, Laughlin, and Conway (1999) tested 133 participants on a diverse battery of STM, WM, and Gf tasks. Consistent with their hypothesis, STM and WM latent variables were separable but correlated .68. In other words, the two-factor model fit the data significantly better than the single-factor model, with a correlation that suggested a close but separable relationship.

Second, if we are correct that controlled attention is important for fluid intelligence, then WM should relate to Gf even after partialling variance due to STM. This prediction is based on the assumption that WM and STM tasks both incorporate domain-specific storage and rehearsal; therefore, partialling STM from WM eliminates primarily domain-specific storage and rehearsal. The residual variance then represents primarily controlled attention, which we argue is important for fluid intelligence. As can be seen from Figure 5.1, even after extracting the variance common to WM and STM, the residual variance in WM correlates with a latent Gf factor at .49, whereas the STM-Gf correlation is nonsignificant. Thus, some aspect of WMC other than task-specific storage processes accounts for the relationship between WMC and Gf variance. Engle, Tuholski, et al. (1999) argued that it was control of attention that mediated the relationship. The controlled attention model (see Engle, Kane, et al., 1999, for a review), by nature, posits a single, domaingeneral WM system, and this seems to fit well with a Gf factor of intelligence.

However, Shah and Miyake (1996) argued for a multiple-resources approach: They found that a verbal working memory measure correlated with verbal Scholastic Aptitude Test ${ }^{3}$ (SAT) performance, whereas a spatial working memory measure did not. Conversely, a spatial working memory span task predicted a spatial reasoning factor, but verbal working memory span did not. Subsequent structural equation models supported their view. In addition, Shah and Miyake crossed the processing and storage 


\section{0 • HANDBOOK OF UNDERSTANDING AND MEASURING INTELLIGENCE}

domains to create four working memory measures, the domains of which either matched or did not match (e.g., spatial WM-spatial reasoning vs. spatial WM-verbal reasoning). The data indicated that it was the storage domain that differentiated predictive ability: WM measures with verbal storage correlated best with verbal reasoning, whereas WM measures with spatial storage correlated best with spatial reasoning.

Although these results are compelling, it is likely that the participant population used by Shah and Miyake (1996) may have restricted the range of abilities tested. They were very likely high in both WMC and Gf. Supporting this, the SAT scores reported for their participants were well above the national average; verbal SAT $M=573$, quantitative SAT $M=655$. At high levels of general ability, most of the variability is accounted for by specific abilities. This situation is similar to what Spearman (1927) called the "law of diminishing returns" and led Jensen (1998) to speculate that, "like money, $g$ isn't very important if one has enough of it" (p. 586). An example of this can be found in Guilford (1979), who argued against the existence of $g$ in favor of a "facet" model consisting of specific abilities. As Stankov (1983) pointed out, Guilford selected mainly individuals of high ability, leading to range restriction and attenuation of variable intercorrelations, thus precluding the emergence of a $g$ factor. For this reason, both the Engle, Tuholski, et al. (1999) and Kane et al. (2003) factor-analytic studies (as well as the experimental studies previously discussed) test a broad range of abilities that include students from a large comprehensive state university and individuals from a diverse metropolitan community.

To address the domain-specific versus domain-general nature of WM, Kane et al. (2003) conducted a large-scale factor analysis that included tasks designed to measure verbal and visuospatial STM and WMC, as well as a battery of fluid intelligence measures, some of which were domain specific (verbal and spatial reasoning) and some of which represented decontextualized reasoning. Kane et al. found that a model with a single WM latent factor fit the data quite well. Although a model with separate verbal and visual WMC factors also fit the data, the two factors correlated .93 , suggesting that a domain-general construct is the most parsimonious explanation of the results. Kane et al. then explored how the single-factor WM model relates to Gf. As illustrated in Figure 5.2, three latent variables were extracted from the reasoning measures. First, a single Gf variable was allowed to load onto all of the reasoning measures. Second, separate verbal and spatial reasoning factors were loaded onto verbal and spatial reasoning tasks, respectively. The WM factor had a strong positive effect on the Gf factor, as well as weak but significant loadings on the domain-specific reasoning factors. Thus, domain-general WMC was found to be important for both general fluid intelligence as well as more specific reasoning factors. In another model (see Figure 5.3), Kane et al. examined how domain-general controlled attention, as well as domain-specific storage, relates to Gf. This analysis reflects our view that WM is composed of both executive attention and storage mechanisms. The "ExecAttn" factor in the model represents the variance shared by WM and STM tasks and, in line with Engle, Tuholski, et al. (1999), reflects primarily executive attention. ${ }^{4}$ This factor was found to have a significant loading on the Gf factor, again about .60 , as well as significant loadings on verbal and spatial reasoning. In addition, the domainspecific storage factors were found to load onto the domain-specific reasoning factors. Consistent with the executive attention theory, the verbal storage factor did not relate to Gf; however, spatial storage did load highly onto Gf, suggesting that spatial tasks, even when they require only passive storage, are related to general fluid intelligence over and above executive attention (see also Chapter 22, this volume).

The above micro- and macroanalytic research makes clear that WMC reflects a domain-general ability to control attention, and it is this same ability that consistently accounts for a substantial amount of the variance in a variety of fluid intelligence measures. It might be straightforward to then conclude that WMC is fluid intelligence. Indeed, this is the conclusion drawn by Kyllonen and Christal (1990). This might be a reasonable deduction, given that they found near-unity correlations between WMC and fluid intelligence. But in most studies, this relationship is appreciably smaller (Ackerman, Beier, \& Boyle, 2002). If WMC 


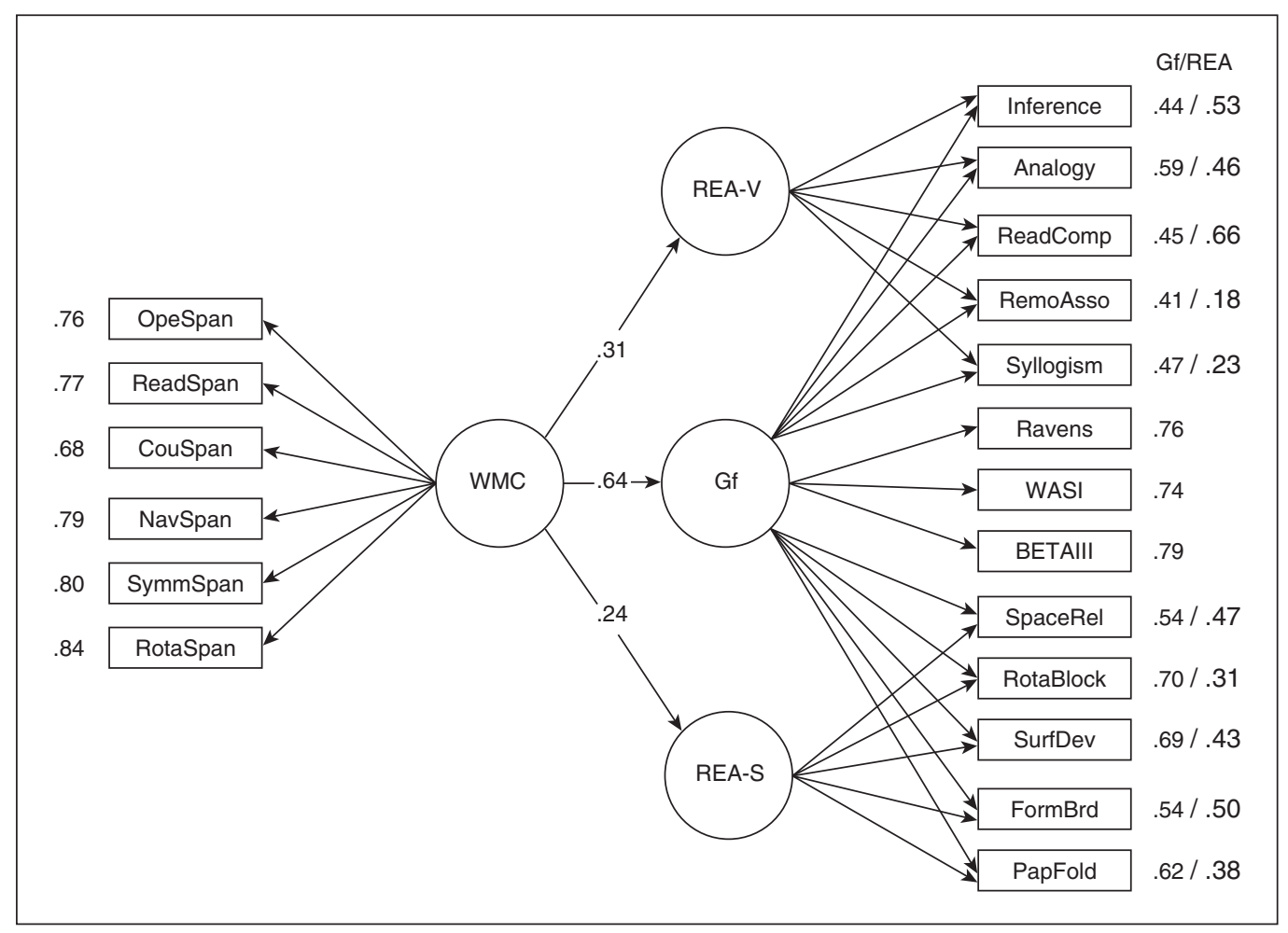

Figure 5.2 Structural Equation Model From Kane et al. (2003)

Note: Boxes represent manifest, or observed, variables; circles represent latent factors. Paths connecting manifest variables represent correlated error terms. All paths are significant at $p<.05$. Numbers under the Gf column represent the loadings of that task on the Gf latent factor (left) and the domain-specific reasoning factor (right). Gf = general fluid intelligence; REA$\mathrm{V}=$ reasoning — verbal; REA-S = reasoning—spatial; OpeSpan = operation span; ReadSpan = reading span; CouSpan = counting span; NavSpan = navigation span; SymmSpan = symmetry span; RotaSpan = rotation span; WordSpan = word span; LettSpan = letter span; DigSpan = digit span; BallSpan = ball span; ArrwSpan = arrow span; MatxSpan = matrix span; Inference = ETS inferences; Analogy $=$ AFOQT analogies; ReadComp $=$ AFOQT reading comprehension; RemoAsso = remote associates; Syllogism = ETS nonsense syllogisms; SpaceRel = DAT space relations; RotaBlock = AFOQT rotated blocks; SurfDev = ETS surface development; FormBrd = ETS form board; PapFold = ETS paper folding; Ravens = Raven's Advanced Progressive Matrices; WASI = Wechsler Abbreviated Scale of Intelligence, Matrix Test; BETAIII = Beta III Matrix Test.

were fluid intelligence, we would expect to find correlations near 1.0 on a consistent basis. We assert that there are many components important to $\mathrm{Gf}$, the most important being controlled attention. However, it is as yet an open question as to why controlled attention is important to Gf. Microanalytic research shows that the ability to control attention is related to both complex cognition involving comprehension, reasoning, and complex learning and very simple cognitive tasks involving primarily attention allocation and control. Macroanalytic research shows that controlled, executive attention accounts for a significant proportion of variance in Gf, even when controlling for domain-specific processes.
However, the specific action of attention on tests such as Raven's remains unclear.

\section{Alternative ExPlanations FOR INDIVIDUAL DIFFERENCES IN INTELLIGENCE}

Our position, that fluid intelligence relies heavily on attentional ability, is certainly not the only explanation for the WMC-intelligence (or attention-intelligence) relationship. Individual differences in basic processing speed (PS) is one alternative theory that has a large base of empirical support: Correlations between processing 


\section{2 • HANDBOOK OF UNDERSTANDING AND MEASURING INTELLIGENCE}

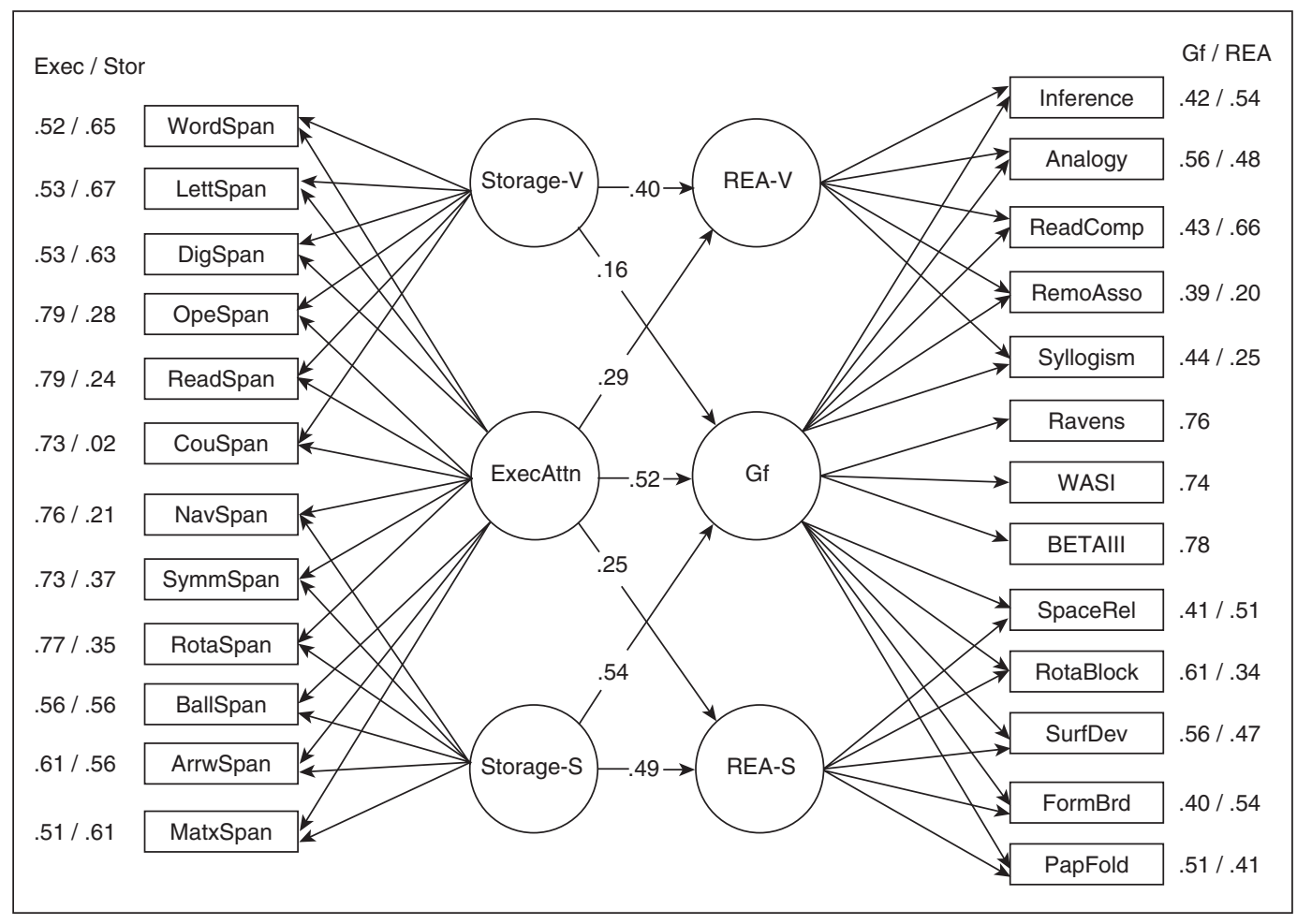

Figure 5.3 Structural Equation Model From Kane et al. (2003)

Note: Boxes represent manifest, or observed, variables; circles represent latent factors. Paths connecting manifest variables represent correlated error terms. All paths are significant at $p<.05$, except the path from Storage- $\mathrm{V}$ to Gf. The numbers under the working memory (WM) column represent the task loadings on the ExecAttn factor, and the numbers under the short-term memory (STM) column represent task loadings on the verbal or spatial STM factor. Numbers under the Gf column represent the loadings of that task on the Gf latent factor (left) and the domain-specific reasoning factor (right). ExecAttn = executive attention; Storage-V = storage—verbal; Storage-S = storage—spatial; Gf = general fluid intelligence; REAV = reasoning — verbal; REA-S = reasoning—spatial; OpeSpan = operation span; ReadSpan = reading span; CouSpan = counting span; NavSpan = navigation span; SymmSpan = symmetry span; RotaSpan $=$ rotation span; WordSpan $=$ word span; LettSpan $=$ letter span; DigSpan = digit span; BallSpan = ball span; ArrwSpan = arrow span; MatxSpan = matrix span; Inference = ETS inferences; Analogy = AFOQT analogies; ReadComp = AFOQT reading comprehension; RemoAsso = remote associates; Syllogism = ETS nonsense syllogisms; SpaceRel $=$ DAT space relations; RotaBlock $=$ AFOQT rotated blocks; SurfDev $=$ ETS surface development; FormBrd = ETS form board; PapFold = ETS paper folding; Ravens = Raven's Advanced Progressive Matrices; WASI $=$ Wechsler Abbreviated Scale of Intelligence, Matrix Test; BETAIII = Beta III Matrix Test.

speed and intelligence are quite reliable (Jensen, 1998; Jensen \& Munro, 1979). But the idea that intellectual abilities are due to PS offers no explanatory mechanism to account for the correlations (see Conway's discussion of "greedy reductionism" in Chapter 4, this volume). In other words, one must wonder, "Speed of what?" Some researchers have speculated that the PS-intelligence relationship reflects "neural efficiency" (e.g., Osmon \& Jackson, 2002), but this then begs the question, "Efficiency of what?" However, there do appear to be at least two views that either propose some mechanism or, at the very least, acknowledge that PS tasks share variance with some other important underlying construct that is a primitive for intellectual functioning.

The first sees intelligence as neural speed. By this view, individual differences in intelligence are due solely to differences in mental speed. This idea proposes that persons who perform mental operations more quickly are likely to have more efficient mental processes and lose less information due to decay over time 
(Reed \& Jensen, 1993; Stankov \& Roberts, 1997; Vernon, 1983). However, a conception such as this requires neurological evidence favoring raw mental speed, otherwise known as nerve conduction velocity (NCV). For example, Reed and Jensen $(1992,1993)$ found significant negative correlations between intelligence measures and latency of the P100 visually evoked potential (using electroencephalograms). But findings such as these are unreliable, are not consistently replicated (e.g., Wickett \& Vernon, 1994), and occasionally are in the opposite direction as would be expected. Stelmack, Knott, and Beauchamp (2003), for example, using brainstem auditory-evoked potentials, observed slower NCVs for individuals of high ability as compared to individuals of low ability. Thus, the literature implicating NCV as a causal mechanism for individual differences in processing speed and intelligence is inconclusive at best.

Developmental studies, which find that processing speed accounts for much of the individual-differences variance in WMC and age, do appear to have some merit. As theories of general slowing make clear, as individuals age, there is a loss of both neural density as well as myelination, which affects NCV (E. M. Miller, 1994). In addition, Salthouse (1992) found that statistically controlling for variability in PS greatly attenuated the relationship between age and WMC. In this case, the PS account may have some validity-older adults experience a decrement in myelinated axons, suggesting that they might suffer greater cross-talk in neural activity and, as a result, may be slower to perform all mental activities (E. M. Miller, 1994). It is also established that the prefrontal cortex, specifically, declines significantly with age; in addition, there is a general reduction in neurotransmitter substance as well as neurotransmitter receptor sites, particularly for dopamine (Rypma, Prabhakaran, Desmond, \& Gabrieli, 2001; West, 1996). It must be noted that although this is a plausible account for the PSintelligence relationship for aging studies, it cannot explain the relationship observed for healthy, young adult populations.

The second view explains processing speed as a variable that taps the true latent construct that accounts for the speed-intelligence correlation. In other words, although it is true that tasks designed to measure processing speed and inspection time consistently load on intelligence factors, these tasks tap some other variable that comprises the major portion of variance in $g$ (e.g., Horn, 1980). We argue that the underlying mechanism is individual differences in the ability to control attention and that there are two mechanisms that bring about the speedintelligence relationship. The first mechanism is lapses in attention, leading to a loss of task goals and productions. Evidence for this claim lies in response time tasks, which find that it is not mean or median RT that correlates best with intelligence measures but instead the standard deviation and skew of the response time distribution (Jensen, 1998; Juhel, 1993; Larson \& Saccuzzo, 1989). Juhel (1993), for example, found a negative correlation between the skew of the RT distribution and intelligence, indicating that individuals of lower ability were also those individuals likely to have unusually long RTs. Often, researchers "trim" the means, removing those specific trials. However, upon closer inspection, it is the long RTs in particular that correlate well with intelligence. Larson and Alderton (1990) rank-ordered RTs from a processing speed task, creating 16 RT bins with equal numbers of observations in each; they then correlated each bin with a $g$ factor. There was a monotonic linear increase in the RT bin-intelligence correlation, as well as an RT bin-working memory correlation. Hence, the differences between high- and low-intelligence individuals are more evident at long RTs, particularly RTs that are slow enough as to be considered lapses in attention. Individuals who are unable to efficiently control their attention when performing a task might be expected to perform in such a manner.

The other mechanism through which attention can mediate the processing speedintelligence relationship is task complexity (e.g., Carpenter, Just, \& Shell, 1990). Conway, Cowan, Bunting, Therriault, and Minkoff (2002) conducted a factor analysis and structural equation model involving STM, WM, and simple PS tasks. Importantly, Conway et al. found that the simple PS tasks did not load on the Gf factor. In other studies (e.g., Ackerman et al., 2002; Babcock, 1994), complex PS tasks are found to 


\section{4 • HANDBOOK OF UNDERSTANDING AND MEASURING INTELLIGENCE}

correlate much more strongly with intelligence and WM than do simple PS tasks. Simply put, PS tasks correlate with intelligence to the extent that they tap attentional ability (Stankov \& Roberts, 1997; Wilhelm \& Schulze, 2002).

\section{Conclusions}

In this chapter, we have reviewed evidence supporting a controlled-attention view of WMC. We argued that the ability to control attention is basic to many real-world phenomena and consistently dissociates people who score high and low on measures of WMC. We also advanced the idea that executive attentional abilities are in some way related to general fluid intelligence. We also make a case against processing speed as an explanatory mechanism for individual differences in WMC and intelligence.

In answer to the question opening this chapter, posed by Spearman in 1927, we would say that attention is partly the same as intelligence. Our program of research makes clear that the ability to control attention is in some way related to fluid intelligence, but it is, as yet, unclear why. In addition, as Ackerman et al. (2002) argue, fluid intelligence and WMC are not isomorphic; correlations range in magnitude (typically from .3 to .6) but are clearly not 1.0 . We rest with the conclusion that of the many systems important for high-level functioning, as might be measured by a Gf measure, attention plays a significant role. The most puzzling realization is that we have good reason to implicate attention in Gf, but we are devoid of a suitable explanation for how attention comes into play when performing a task such as the Raven. Further research in our lab and, it is hoped, in others aims to elucidate this issue.

\section{Notes}

1. The operation span task demonstrates good test-retest and internal validity (Engle, Tuholski, Laughlin, \& Conway, 1999; Klein \& Fiss, 1999).

2. Interestingly, across three experiments and an additional control experiment, high spans were found to have reliably larger baseline pupil sizes not only during the task but also before the task even began.
3. The Scholastic Aptitude Test, or SAT, is a comprehensive achievement test that is required for admission to most American colleges.

4. It should be noted that although they appear similar, the "common" factor extracted in the Engle, Tuholski, et al. (1999) paper and the "ExecAttn" factor extracted in the Kane et al. (2003) paper are not identical. Engle, Tuholski, et al. (1999) incorporated short-term memory (STM) tasks that were primarily verbal in nature. If we assume that STM = verbal ability and working memory $(\mathrm{WM})=$ verbal ability + controlled attention, the "Common" factor contains only variance due to verbal ability, leaving executive attention as residual. Kane et al., on the other hand, made use of a wider variety of WM and STM tasks. Critically, some of these tasks were spatial, whereas some were verbal. We assume that working memory capacity (WMC) tasks measure executive attention primarily but also some domain-specific skills. Likewise, we assume that STM tasks measure domain-specific skills primarily but also some executive attention. Therefore, when Kane et al. extracted variance common to all of the tasks, the factor comprised only executive attention. In other words, verbal tasks (both STM and WMC variants) consist of verbal skills and executive attention. Spatial tasks (again both STM and WMC variants) consist of spatial skills and executive attention. Thus, the common variance between the two is executive attention.

\section{REFERENCES}

Ackerman, P. L., Beier, M. E., \& Boyle, M. O. (2002). Individual differences in working memory within a nomological network of cognitive and perceptual speed abilities. Journal of Experimental Psychology: General, 131, 567-589.

Ahern, S. K., \& Beatty, J. (1979). Pupillary responses during information processing vary with Scholastic Aptitude Test scores. Science, 205, 1289-1292.

Anderson, J. R., Reder, L. M., \& Lebiere, C. (1996). Working memory: Activation limitations on retrieval. Cognitive Psychology, 30, 221-256.

Atkinson, R. C., \& Shiffrin, R. M. (1968). Human memory: A proposed system and its control processes. In K. W. Spence \& J. T. Spence (Eds.), The psychology of learning and motivation (Vol. 2, pp. 89-195). New York: Academic Press. 
Babcock, R. L. (1994). Analysis of adult age differences on the Raven Advanced Progressive Matrices test. Psychology and Aging, 9, 303-314.

Baddeley, A. D. (1996). Exploring the central executive. Quarterly Journal of Experimental Psychology, 49A, 5-28.

Binet, A. (1903). L'étude expérimentale de l'intelligence [The experimental study of intelligence]. Paris: Schleicher Frères.

Brewin, C. R., \& Beaton, A. (2002). Thought suppression, intelligence, and working memory capacity. Behavior Research and Therapy, 40, 923-930.

Burt, C. (1909). Experimental tests of general intelligence. British Journal of Psychology, 3, 94-177.

Cantor, J., \& Engle, R. W. (1993). Working memory capacity as long-term memory activation: An individual differences approach. Journal of Experimental Psychology: Learning, Memory and Cognition, 19, 1101-1114.

Carpenter, P. A., Just, M. A., \& Shell, P. (1990). What one intelligence test measures: A theoretical account of the processing in the Raven Progressive Matrices test. Psychological Review, 97, 404-431.

Carroll, J. B. (1993). Human cognitive abilities: A survey of factor-analytic studies. New York: Cambridge University Press.

Cattell, R. B. (1943). The measurement of adult intelligence. Psychological Bulletin, 40, 153-193.

Cattell, R. B. (1963). Theory of fluid and crystallized intelligence: A critical experiment. Journal of Educational Psychology, 54, 1-22.

Cherry, E. C. (1953). Experiments on the recognition of speech with one and two ears. Journal of the Acoustical Society of America, 25, 975-979.

Conway, A. R. A., Cowan, N., \& Bunting, M. F. (2001). The cocktail party phenomenon revisited: The importance of working memory capacity. Psychonomic Bulletin \& Review, 8, 331-335.

Conway, A. R. A., Cowan, N., Bunting, M. F., Therriault, D. J., \& Minkoff, S. R. B. (2002). A latent variable analysis of working memory capacity, short-term memory capacity, processing speed, and general fluid intelligence. Intelligence, 30, 163-183.

Conway, A. R. A., \& Engle, R. W. (1994). Working memory and retrieval: A resource-dependent inhibition model. Journal of Experimental Psychology: General, 123, 354-373.
Daneman, M., \& Carpenter, P. (1980). Individual differences in working memory and reading. Journal of Verbal Learning and Verbal Behavior, 19, 450-466.

Daneman, M., \& Carpenter, P. (1983). Individual differences in integrating information between and within sentences. Journal of Experimental Psychology: Learning, Memory, and Cognition, 9, 561-584.

Daneman, M., \& Tardif, T. (1987). Working memory and reading skill re-examined. In M. Coltheart (Ed.), Attention and performance XII (pp. 491508). Hillsdale, NJ: Lawrence Erlbaum.

De Jong, R., Berendsen, E., \& Cools, R. (1999). Goal neglect and inhibitory limitations: dissociable causes of interference effects in conflict situations. Acta Psychologica, 101, 379-394.

D’Esposito, M., Detre, J. A., Alsop, D. C., \& Shin, R. K. (1995). The neural basis of the central executive system of working memory. Nature, 378, 279-281.

Engle, R. W., Cantor, J., \& Carullo, J. (1992). Individual differences in working memory and comprehension: A test of four hypotheses. Journal of Experimental Psychology: Learning, Memory and Cognition, 18, 972-992.

Engle, R. W., Kane, M. J., \& Tuholski, S. W. (1999). Individual differences in working memory capacity and what they tell us about controlled attention, general fluid intelligence, and functions of the prefrontal cortex. In A. Miyake \& P. Shah (Eds.), Models of working memory: Mechanisms of active maintenance and executive control (pp. 102-134). Cambridge, UK: Cambridge University Press.

Engle, R. W., Nations, J. K., \& Cantor, J. (1990). Word knowledge and working memory capacity. Journal of Educational Psychology, 82, 799-804.

Engle, R. W., Tuholski, S. W., Laughlin, J. E., \& Conway, A. R. A. (1999). Working memory, shortterm memory and general fluid intelligence: A latent variable approach. Journal of Experimental Psychology: General, 128, 309-331.

Fogarty, G., \& Stankov, L. (1982). Competing tasks as an index of intelligence. Personality and Individual Differences, 3, 407-422.

Guilford, J. P. (1979). Intelligence isn't what it used to be: What to do about it. Journal of Research and Development in Education, 12, 33-44.

Hasher, L., \& Zacks, R. T. (1988). Working memory, comprehension, and aging: A review and a new 


\section{6 • HANDBOOK OF UNDERSTANDING AND MEASURING INTELLIGENCE}

view. In G. H. Bower (Ed.), The psychology of learning and motivation (Vol. 22, pp. 193-225). New York: Academic Press.

Heitz, R. P., Schrock, J. C., Payne, T. W., \& Engle, R. W. (2003). Working memory and mental effort: The eyes have it. Manuscript submitted for publication.

Hess, E. H., \& Polt, J. M. (1964). Pupil size in relation to mental activity during simple problemsolving. Science, 143, 1190-1192.

Horn, J. L. (1980). Concepts of intellect in relation to learning and adult development. Intelligence, 4 , 285-317.

James, W. (1981). The principles of psychology (Vol. 1). Cambridge, MA: Harvard University Press. (Original work published 1890)

Jensen, A. R. (1998). The g factor. Westport, CT: Praeger.

Jensen, A. R., \& Munro, E. (1979). Reaction time, movement time, and intelligence. Intelligence, 3, 121-126.

Juhel, J. (1993). Should we take the shape of reaction time distributions into account when studying the relationship between RT and psychometric intelligence? Personality and Individual Differences, 15, 357-360.

Kahneman, D., \& Beatty, J. (1966). Pupil diameter and load on memory. Science, 154, 1583-1585.

Kane, M. J., Bleckley, K. M., Conway, A. R. A., \& Engle, R. W. (2001). A controlled-attention view of working-memory capacity. Journal of Experimental Psychology: General, 130, 169-183.

Kane, M. J., \& Engle, R. W. (2000). Working-memory capacity, proactive interference, and divided attention: Limits on long-term memory retrieval. Journal of Experimental Psychology: Learning, Memory, \& Cognition, 26, 336-358.

Kane, M. J., \& Engle, R. W. (2002). The role of prefrontal cortex in working-memory capacity, executive attention, and general fluid intelligence: An individual differences perspective. Psychonomic Bulletin \& Review, 9, 637-671.

Kane, M. J., Hambrick, D. Z., Tuholski, S. W., Wilhelm, O., Payne, T. W., \& Engle, R. W. (2003). The generality of working-memory capacity: A latent-variable approach to verbal and visuo-spatial memory span and reasoning. Manuscript submitted for publication.

Klein, K., \& Fiss, W. H. (1999). The reliability and stability of the Turner and Engle working memory task. Behavioral Research Methods, Instruments, \& Computers, 31, 429-432.
Kyllonen, P. C., \& Christal, R. E. (1990). Reasoning ability is (little more than) working-memory capacity?! Intelligence, 14, 389-433.

Larson, G. E., \& Alderton, D. L. (1990). Reaction time variability and intelligence: A "worst performance" analysis of individual differences. Intelligence, 14, 309-325.

Larson, G. E., \& Saccuzzo, D. P. (1989). Cognitive correlates of general intelligence: Toward a process theory of $g$. Intelligence, 13, 5-31.

Logan, G. D., \& Zbrodoff, N. J. (1979). When it helps to be misled: Facilitative effects of increasing the frequency of conflicting stimuli in a Strooplike task. Memory \& Cognition, 7, 166-174.

Marshalek, B., Lohman, D. F., \& Snow, R. E. (1983). The complexity continuum in the radex and hierarchical models of intelligence. Intelligence, 7, 107-127.

Miller, G. A. (1956). The magical number seven, plus or minus two: Some limits on our capacity for processing information. Psychological Review, 63, 81-97.

Miller, E. M. (1994). Intelligence and brain myelination: A hypothesis. Personality and Individual Differences, 17, 803-832.

Moray, N. (1959). Attention in dichotic listening: Affective cues and the influence of instructions. Quarterly Journal of Experimental Psychology, 11, 56-60.

Osmon, D. C., \& Jackson, R. (2002). Inspection time and fluid IQ: Fluid or perceptual aspects of intelligence? Intelligence, 30, 119-128.

Parasuraman, R. (Ed.). (1998). The attentive brain. Cambridge: MIT Press.

Reed, T. E., \& Jensen, A. R. (1992). Conduction velocity in a brain nerve pathway of normal adults correlates with intelligence level. Intelligence, 16, 259-272.

Reed, T. E., \& Jensen, A. R. (1993). Choice reaction time and visual pathway nerve conduction velocity both correlate with intelligence but appear not to correlate with each other: Implications for information processing. Intelligence, 17, 191-203.

Rosen, V. M., \& Engle, R. W. (1997). The role of working memory capacity in retrieval. Journal of Experimental Psychology: General, 126, 211-227.

Rosen, V. M., \& Engle, R. W. (1998). Working memory capacity and suppression. Journal of Memory and Language, 39, 418-436. 
Rypma, B., Prabhakaran, V., Desmond, J. E., \& Gabrieli, J. D. E. (2001). Age differences in prefrontal cortical activity in working memory. Psychology and Aging, 16, 371-384.

Salthouse, T. A. (1992). Influence of processing speed on adult age differences in working memory. Acta Psychologica, 79, 155-170.

Schneider, W., \& Shiffrin, R. M. (1977). Controlled and automatic human information processing: I. Detection, search, and attention. Psychological Review, 84, 1-66.

Shah, P., \& Miyake, A. (1996). The separability of working memory resources for spatial thinking and language processing: An individual differences approach. Journal of Experimental Psychology: General, 125, 4-27.

Spearman, C. (1904). 'General intelligence,' objectively determined and measured. American Journal of Psychology, 15, 201-293.

Spearman, C. (1927). The abilities of man. London: Macmillan.

Stankov, L. (1983). Attention and intelligence. Journal of Educational Psychology, 75, 471-490.

Stankov, L. (1989). Attentional resources and intelligence: A disappearing link. Personality and Individual Differences, 10, 957-968.

Stankov, L., \& Roberts, R. D. (1997). Mental speed is not the 'basic' process of intelligence. Personality and Individual Differences, 22, 69-84.

Stelmack, R. M., Knott, V., \& Beauchamp, C. M. (2003). Intelligence and neural transmission time: A brain stem auditory evoked potential analysis. Personality and Individual Differences, 34, 97-107.
Sternberg, R. J. (1982). Handbook of human intelligence. New York: Cambridge University Press.

Sternberg, R. J. (1985). Beyond IQ: A triarchic theory of human intelligence. New York: Cambridge University Press.

Thurstone, L. L. (1938). Primary mental abilities. Psychometric Monographs, 1, 1-121.

Tuholski, S. W., Engle, R. W., \& Baylis, G. C. (2001). Individual differences in working memory capacity and enumeration. Memory \& Cognition, 29, 484-492.

Turner, M. L., \& Engle, R. W. (1989). Working memory capacity: An individual differences approach. Journal of Memory and Language, 28, 127-154.

Unsworth, N., Schrock, J. C., \& Engle, R. W. (2003). Working memory capacity and the antisaccade. Manuscript in preparation.

Vernon, P. A. (1983). Speed of information processing and general intelligence. Intelligence, 7, 53-70.

West, R. L. (1996). An application of prefrontal cortex function theory to cognitive aging. Psychological Bulletin, 120, 272-292.

Wickett, J. C., \& Vernon, P. A. (1994). Peripheral nerve conduction velocity, reaction time, and intelligence: An attempt to replicate Vernon and Mori (1992). Intelligence, 18, 127-131.

Wilhelm, O., \& Schulze, R. (2002). The relation of speeded and unspeeded reasoning with mental speed. Intelligence, 30, 537-554.

Wood, N., \& Cowan, N. (1995). The cocktail party phenomenon revisited: How frequent are attention shifts to one's name in an irrelevant auditory channel? Journal of Experimental Psychology: Learning, Memory, and Cognition, 21, 255-260. 
05-Wilhelm.qxd 7/21/2004 7:39 PM Page 78 